\title{
Mass spectrometry based proteomic analysis of human stem cells: a brief review
}

\author{
Moon-Young Choi ${ }^{1}$, Yoo Jin $\mathrm{An}^{1}$, So Hyun Kim ${ }^{1}$, \\ Si Hun Roh', Hyun Kyung Ju', Soon Sun Hong', \\ Jeong Hill Park ${ }^{1}$, Kyoung Jin $\mathrm{Cho}^{3}$, \\ Dal Woong Choi, ${ }^{3,4}$ and Sung Won Kwon ${ }^{1}$ \\ ${ }^{1}$ College of Pharmacy \\ Seoul National University \\ Seoul 151-742, Korea \\ ${ }^{2}$ College of Medicine, Inha University \\ Incheon 402-751, Korea \\ ${ }^{3}$ College of Health Sciences \\ Korea University \\ Seoul 136-703, Korea \\ ${ }^{4}$ Corresponding author: Tel, 82-11-9115-7875; \\ Fax, 82-2-940-2861; E-mail, aahhhh@paran.com
}

Accepted 29 October 2007

\begin{abstract}
Abbreviations: 2-DE, two-dimensional electrophoresis; BM, bone marrow; EGF, embryonic growth factor; FGF, fibroblast growth factor; hESCs, human embryonic stem cells; hMSCs, human mesenchymal stem cells; hNSCs, human neuronal stem cells; ICAT, isotope-coded affinity tag; iTRAQ, isobaric tags for relative and absolute quantification; LC/MS/MS, liquid chromatography tandem mass spectrometry; MALDI-TOF, matrix assisted laser desorption/ ionization time-of-flight; MPCs, mesenchymal progenitor cells; MSCs, mesenchymal stem cells; PCNA, proliferating cell nuclear antigen; PIP2, phosphatidylinositol bisphosphate; PMF, peptide mass fingerprinting; pRB, retinoblastoma protein; PRDX 1, peroxiredoxin 1; PRDX 4, peroxiredoxin 4; PTM, posttranslational modification; SELDI-TOF, surface enhanced laser desorption/ ionization time-of-flight; SILAC, stable isotope labeling by amino acids in cell culture; SPITC, 4-sulfophenyl isothiocyanate; TAGL2, transgelin-2; TCTP, translationally controlled tumor protein; UCB, umbilical cord blood
\end{abstract}

\begin{abstract}
Stem cells can give rise to various cell types and are capable of regenerating themselves over multiple cell divisions. Pluripotency and self-renewal potential of stem cells have drawn vast interest from different disciplines, with studies on the molecular properties of stem cells being one example. Current investigations on the molecular basis of stem cells pluripotency and self-renewal entail traditional techniques from chemistry and molecular biology. In this mini review, we discuss progress in stem cell research that employs pro-
\end{abstract}

teomics approaches. Specifically, we focus on studies on human stem cells from proteomics perspective. To our best knowledge, only the following types of human stem cells have been examined via proteomics analysis: human neuronal stem cells, human mesenchymal stem cells, and human embryonic stem cells. Protein expression serves as biomarkers of stem cells and identification and expression level of such biomarkers are usually determined using two-dimensional electrophoresis coupled mass spectrometry or non-gel based mass spectrometry.

Keywords: adipocytes; electrophoresis, gel, two-dimensional; embryonic stem cells; human neuronal stem cells; mass spectrometry; mesenchymal stem cells; proteomics

\section{Introduction}

Stem cells have various unique characteristics; they can divide and renew themselves over many generations and have multi-potentiality. Differentiation and maturation of stem cells comprises complex serial events, which guide the undifferentiated cells to different lineages via distinctive developmental programs (Chung et al., 2005; Hoffrogge et al., 2006; Kwak et al., 2006). Such regulatory changes can be studied systematically with transgenic technology and microarrays (Pazman et al., 2000; Böttcher et al., 2003; Gurok et al., 2004; Pahnke et al., 2004). The process of differentiation entails changes in types and amount of proteins expressed by the stem cell. Therefore, protein expression pattern can provide important clues about the progression differentiation process for stem cells at various stages.

Proteomics, the large-scale study of proteins, holds great promise in unraveling the molecular basis of stem cell differentiation. Proteomics has emerged as a robust method for comparing protein expression under different conditions that supplements high-throughput gene expression analysis at the RNA level (Baharvand et al., 2006b). Proteomics approaches have been applied to create the molecular map of protein expression in stem cells. Proteome maps, or profiling of proteomes, have greatly contributed to the current body of knowledge on the biomarkers of stem cells. Functional proteomic analysis at a certain biological system 
requires global quantification of all the protein machinery (Ma et al., 2007).

Early efforts on stem cell proteomics heavily relied on two-dimensional electrophoresis (2-DE) and MS analysis for protein identification. Nowadays, several new techniques empowered with both qualitative and quantitative aspects are available: chemical isotope tagging techniques to isotope-coded affinity tag (ICAT), isobaric tags for relative and absolute quantification (iTRAQ), and 4-sulfophenyl isothiocyanate (SPITC) (Gygi et al., 1999; Lee et al., 2004; Ross et al., 2004). In addition, all-around enzymatic isotope labeling techniques have also been applied, such as stable isotope labeling by amino acids in cell culture (SILAC) for in vivo labeling (Ong et al., 2002), and enzymatic labeling of ${ }^{18} \mathrm{O}$ to C-terminal of peptides by proteases in the presence of ${ }^{18} \mathrm{O}$ water $\left(\mathrm{H}_{2}{ }^{18} \mathrm{O}\right)$ (Stewart et al., 2001).

Issues such as loss of 3-D structure information upon linearization of proteins, incomplete posttranslational modification mapping, and concentration dynamics problem due to lack of effective analyte enrichment technology (Aebersold and Cravatt, 2002; Hood, 2003) pose challenges to proteomics. Proteomics analysis should overcome the hurdles ahead in order to clear itself of the charge of being the bottleneck for systems-level analysis of biological information hierarchy. Nevertheless, to date current state-of-art proteomics approaches provide powerful tools for investigating the molecular basis of stem cell differentiation.

\section{Human neuronal stem cells}

Human neural stem cells (hNSCs) are self-renewing and multipotent precursors that can lead to neuronal and glial progenitor cell, which is in order to differentiate into neurons and astrocytes or oligodendrocytes, respectively (Ma et al., 2007). The first proteomics studies based on 2-DE gel methodology have been reported in 1999, where Pearce and Svendsen (1999) showed first differential 2-DE gel approach to fetal human brain cells treated with both embryonic growth factor (EGF) and fibroblast growth factor (FGF). After Pearce and Svendsen's pioneering work, proteomic profiling has mainly been applied for compiling protein inventory in the adult nervous system of normal versus diseased individuals (Rohlff, 2000, 2001; Husi and Grant, 2001; Lubec et al., 2003; Choudhary and Grant, 2004; Kim et al., 2004; Taylor et al., 2004). However, protein analysis of human fetal neuronal stem cell differentiation has not much progressed yet. Recently proteomics analysis on hNSC was carried out by Hoffrogge et al. (2006). hNSCs differentiated upon removal of growth factors from culture medium, evidenced by clear morphological changes. $500 \mu \mathrm{g}$ of protein samples were loaded onto 2D SDS PAGE gels (25 $\mathrm{cm} \times 22.5 \mathrm{~cm} \times 0.5 \mathrm{~cm}$ ) and stained with colloidal coomassie blue. After in-gel digestion peptide mixtures were analyzed by MALDI-TOF MS (Reflex III mass spectrometer, Bruker Daltonics) and MALDITOF MS/MS (4700 proteomics analyzer, Applied Biosystems) and protein identification searches were performed by MASCOT search engine (Matrix Science).

956 spots were excised from 2-DE gel slab and 412 spots were identified by MALDI-TOF MS. Specific functions of the identified proteins were inferred from the gene ontology database. Circa $21 \%$ of the proteins were related to protein synthesis, metabolism, processing and degradation; less than $11 \%$ were related to cytoskeleton proteins, stress response proteins, the functional group of RNA and other nucleic acids metabolisms and transport, signal transduction and others (Hoffrogge et al., 2006).

According to Hoffrogge et al. (2006) peroxiredoxin 1 (PRDX 1) and transgelin-2 (TAGL2) were up-regulated and proliferating cell nuclear antigen (PCNA) and peroxiredoxin 4 (PRDX 4) were down-regulated in stem cells upon differentiation. PRDX 1 belongs to a family of anti-oxidative proteins and is known to be overexpressed when cells are exposed to proliferative signals or oxidative stress (Prosperi et al., 1998). Transgelin2 , a protein of the calponin family, is the second protein whose expression level increased upon differentiation of hNSCs. Transgelin has direct interaction with actin filaments; saturation of transgelin-binding occurs at the transgelin/actin monomers ratio of 1:6, which causes rapid gelation of transgelin-actin complexes within min (Shapland et al., 1993).

A down-regulated protein, PCNA is an autoantibody to a nuclear antigen in proliferating cells (Miyachi et al., 2005) and correlates to the proliferative status of cells (Takasaki et al., 1984). PRDX 4 is related with NF- $\kappa B$ activation. CrowlyWeber et al. (2002) has found earlier that NF-кB, PRDX 4 and Grp 78 are up-regulated in apoptosis resistant cells. Decreased PRDX4 levels in differentiated NSC may indicate that a pro-apoptotic situation is supported during differentiation. 


\section{Human mesenchymal stem cells}

\section{Human bone marrow stromal cell}

Human bone marrow stromal cells, also known as human mesenchymal stem cells (hMSCs) or mesenchymal progenitor cells (MPCs), are the main element of the bone marrow microenvironment and support in promoting differentiation and proliferation of hematopoietic cells. hMSCs are characterized by their high plasticity, which is reflected in the manifold changes in transcription level and protein expression of these cells as they follow different lineages. Thus, proteome profiling of bone marrow stromal cells may provide important insights into the mechanism of normal and dysregulated hematopoiesis, as it was demonstrated in Seshi's work (2006). The proteome of hMPCs from normal versus leukemic individuals was analyzed using iTRAQ technology and 2D LC/MS/MS. $400 \mu \mathrm{g}$ of protein sample was ITRAQ labeled for quantification and analyzed using 2D LC/MS/MS (2D LC; LC Packing, MS/MS; QStar Pulsar i, Applied Biosystems). For protein identification and quantification Pro Quant software (Applied Biosystems MDS SCIEX) was used. 73 out of approximately 900 proteins were differentially expressed in leukemic stromal cells compared with hMPCs.

\section{Human mesenchymal stem cells derived from umbilical cord blood}

While mesenchymal stem cells (MSCs) possess the desirable characteristics of stem cells, they are easy to obtain and culture, making these cells attractive candidates for tissue engineering approaches in mesenchymal tissue regeneration (Feldmann et al., 2005). Their main resource is the bone marrow (BM). BM-MSCs exhibit multipotent differentiation potential and can induce mesodermal originated tissue. However, several complications arise when MSCs are obtained from the bone marrow: the invasive collection procedure, high chance of viral exposure (Amos and Gordon, 1995), sub-optimal proliferation rate in vitro condition, and limited differentiation capacity over multiple passages in tissue culture (Mueller and Glowacki, 2001; Stenderup et al., 2003). Human umbilical cord blood (UCB) is an alternative source of hMSCs that is relatively free of such disadvantages.

Feldmann et al. (2005) compared the difference between BM-MSCs and UCB-MSCs. Immunological phenotype determined by surface proteins of UCB-MSCs was analyzed using FACS flow cytometry. The results confirmed that BM-MSCs and UCB-MSCs displayed same phenotype. As a further investigation on UCB-MSCs, cytosolic protein expression was analyzed via 2-DE. $500 \mu \mathrm{g}$ of protein samples were loaded onto 2D SDS PAGE gels $(20 \mathrm{~cm} \times 18 \mathrm{~cm} \times 0.4 \mathrm{~cm})$ and visualized by silver staining. After automated spot excision by Flexys (Genomic Solutions) and in-gel digestion peptide mixtures were analyzed by MALDI-TOF/TOF MS (Ultraflex TOF/TOF, Bruker Daltonics) and MASCOT query (Matrix Science) was used for protein identification.

As a result, 2037 spots were excised from 2-DE gel slab and 205 spots were identified by MALDITOF-MS. Cellular functions of the resolved proteins were identified using the gene ontology database. The major group consisted of proteins that belong to cellular metabolism. The metabolic protein group encompassed numerous biochemical pathways such as Krebs cycle, amino acid metabolism, cellular housekeeping, and protein biosynthesis. Feldmann et al. (2005) identified the presence of protein vimentin in differentiating stem cells, which is normally absent in undifferentiated forms. Vimentin is the most omnipresent intermediate filament protein and is one of the first proteins to be expressed during the differentiation of the cell. Gelsolin was another one to be identified in differentiating MSCs. Gelsolin is an actin binding protein and displays biphasic responses. Under low calcium concentrations, gelsolin induces nucleation actin polymerization; however, gelsolin causes severing of actin filaments at high calcium concentrations. Gelsolin also binds phosphatidylinositol bisphosphate $\left(\mathrm{PIP}_{2}\right)$, a membrane-bound molecule, thereby functioning as an anchor to actin organization and signal transduction. Gelsolin expression is down-regulated as stem cell is under differentiation (Wang et al., 2004). Such downregulation may also affect cell shape and migratory capacity, and potentially the homing of stem cells (Evans et al., 2004). The protein prohibitin has been known for key functions in progenitor cells; two prohibitin proteins, Phb1p and Phb2p (BAP37), are involved in various functions such as cell cycle regulation, mitosis and proliferation, apoptosis, assembly of mitochondrial respiratory chain enzymes, and aging. Since prohibitins participate in G1/S phase regulation in cell cycle and interact with the retinoblastoma protein (pRB), prohibitins are considered as latent tumor suppressors (Feldmann et al., 2005).

\section{Adipocyte differentiated from human mesenchymal stem cells}

Adipocytes are cells from connective tissues that 
have been characterized in the synthesis and storage of fat. Adipocytes serve important roles in mammalian physiology: maintenance of proper energy balance, storage of energy in lipid forms, mobilization of energy sources in response to hormonal stimulation, and signal transduction (Camp et al., 2002). Better information regarding the molecular events in innate adipocyte differentiation will obviously enhance the treatment of adipocyte-related metabolic diseases such as obesity and diabetes (Nuttall and Gimble, 2000; Sekiya et al., 2004).

Lee et al. (2006) have investigated the protein expression inherent to adipogenic differentiation using 2-DE, MALDI-TOF, and RT-PCR. For the 2-DE and MALDI-TOF, $90 \mu \mathrm{g}$ of protein samples were loaded onto 2D SDS PAGE gels $(26 \mathrm{~cm} \times 20$ $\mathrm{cm}$ ) and visualized with silver staining. After in-gel digestion and desalting with Ziptip C18 (Millipore Corp.), peptide mixtures were analyzed by MALDI-TOF MS (Voyager DESTR mass spectrometer, Applied Biosystems), and protein identification searches were performed by Ms-Fit (http:// prospector.ucsf.edu). The study focused on differential proteome expression in hMSCs versus adipocytes. Lee et al. (2006) performed 2-DE analysis for the cytosolic proteins extracted from both hMSCs and adipocytes differentiated from hMSC. Thirty-two protein spots showed differential proteomic results. Eight proteins were confirmed to be up-regulated by MALDI-TOF/MS: sintaxin binding protein 3, OSBP-related 3, phosphodiesterase, glycophorin, immunoglobulin kappa chain variable region, peroxisome proliferative activated receptor gamma, bA528A10.3.1, and T cell receptor $\mathrm{V}-\beta 4$. Four proteins (syntaxin-3, OSBP-related protein 3 , PPAR- $\gamma$ and glycophorin) were shown to be related with adipogenesis. The differentially expressed proteins between hMSCs and adipocytes might be considered as cytosolic biomarker proteins for adipogenesis (Lee et al., 2006).

\section{Human embryonic stem cells}

Human embryonic stem cells (hESC) consist of a population of undifferentiated pluripotent cells with both self-renewal and multi-lineage differentiation characteristics (Baharvand et al., 2006b). Hayman and Przyborski (2004) reported surface enhanced laser desorption/ionization time-of-flight mass spectrometry (SELDI-TOF-MS) technology to explore potential biomarkers of human embryonal carcinoma (EC) stem cells. They identified prominent biomarkers that showed unique expression pattern in pre-versus post-differentiation human EC cells.
Markers that were constitutively expressed in both populations were also investigated.

Also, Baharvand et al. (2006b) have applied 2-DE and MALDI TOF-TOF MS/MS (4700 Proteomics Analyzer, Applied Biosystems) to profile global map of proteome expressed in hESC. Cell line samples were analyzed in triplicate using 2-DE and 1,250 \pm 150 spots were detected. For MS analysis, $120 \mu \mathrm{g}$ of protein samples were loaded onto 2D SDS PAGE gels (24 cm strips) and stained with colloidal CBB G 250. 844 spots were collected for mass spectrometry based proteomic analysis and 685 proteins were identified by combining peptide mass fingerprinting (PMF) and tandem mass spectral data. Out of 685 proteins 434 proteins corresponded to unique proteins and 251 proteins corresponded to isoforms or posttranslational modification (PTM). There are other reports that have also profiled plenty of isoforms or PTM (Maurer et al., 2003; Elliott et al., 2004). Careful analysis on all isoforms of a given protein is very important because many of the interesting regulatory steps, especially those involved in cell proliferation, migration and differentiation, count on protein PTM more than they do on protein expression levels (Levchenko, 2005). Baharvand et al. (2006b) identified a large number of proteins involved in protein synthesis, processing, and trafficking. Such finding suggests that hESCs are able to maintain the undifferentiated state until signals of lineage determination is received, upon which these cells quickly change phenotype and produce necessary proteins. Seven out of sixty proteins were assigned as up-regulated genes in transcriptomic analysis of hESC: cofilin1, DJ-1 protein, translationally controlled tumorprotein (TCTP), enolase 1 , heat shock congnate $71-\mathrm{kDa}$ protein, latate dehyrogenase $\mathrm{B}$, and necleophosmin 1 (Baharvand et al., 2006b). A large number of proteins, particularly the highly abundant ones, were identified as chaperones, heat shock proteins, ubiquitin/proteasome, and oxidative stress responsive proteins; expression of such proteins at high level implies the ability of hESCs to resist oxidative stress and increase life span. Several proteins involved in cell proliferation and differentiation belonged to the group of up-regulated proteins. Baharvand et al. (2006b) further identified 30 proteins among 1010 mESC- and stemnessspecific proteins identified by Nagano et al. (2005). Baharvand et al. (2006a) have successfully identified the following proteins that may be able to serve as SC-specific biomarkers: hepatoma-derived growth factor, guanine nucleotide-binding protein beta, and CRABP1. However, the authors were unable to detect the products of many 
transcripts that are characteristic to hESC, due to technical limitation of 2-D SDS-PAGE based systems and the discrepancy between the cellular mRNA levels versus protein levels in eukaryotic cells. 2-DE based analysis is obviously confined to examining only the highly abundant and hydrophilic proteins. Furthermore, silver staining method may not be the optimal method to prepare 2-DE gels for MS, especially when the coloration is saturated. Moreover, purifying SC to absolute homogeneity is beyond the limit of current technology.

\section{Discussion}

Stem cells are capable of both self-renewing process and giving rise to diverse tissue types. There is increasing public interest on clinical trial of stem cells. Proteomics, large scale studies of proteins, can be used to analyze protein contents of stem cells in a cost-effective and efficient way. Through 2-DE coupled mass spectrometry or non-gel based mass spectrometry, the basic analytical tools in proteomics, proteins of several types of stem cells such as hNSCs, hMSCs, and hESC have been analyzed. These works using proteomics approach is, however, limited to analyzing highly abundant proteins only. Therefore, new mass spectrometry based proteomic analysis techniques for stem cell proteins must be developed in correlation with other state-of-the-art analytical tools.

\section{Acknowledgement}

This work was supported by grant no. R01-2006-00010593-0 from the Basic Research Program of the Korea Science and Engineering Foundation.

\section{References}

Aebersold R, Cravatt BF. Proteomics--advances, applications and the challenges that remain. Trends Biotechnol 2002;20:S1-2

Amos TA, Gordon MY. Sources of human hematopoietic stem cells for transplantation. Cell Transplant 1995;4:547-69

Baharvand H, Ashtiani SK, Taee A, Massumi M, Valojerdi MR, Yazdi PE, Moradi SZ, Farrokhi A. Generation of new human embryonic stem cell lines with diploid and triploid karyotypes. Dev Growth Differ 2006a;48:117-28

Baharvand H, Hajheidari M, Ashtiani SK, Salekdeh GH. Proteomic signature of human embryonic stem cells. Proteomics 2006b;6:3544-49

Bottcher T, Mix E, Koczan D, Bauer P, Pahnke J, Peters S, Weinelt S, Knoblich R, Strauss U, Cattaneo E, Thiesen HJ,
Rolfs A. Gene expression profiling of ciliary neurotrophic factor-overexpressing rat striatal progenitor cells (ST14A) indicates improved stress response during the early stage of differentiation. J Neurosci Res 2003;73:42-53

Camp HS, Ren D, Leff T. Adipogenesis and fat-cell function in obesity and diabetes. Trends Mol Med 2002;8:442-7

Choudhary J, Grant SG. Proteomics in postgenomic neuroscience: the end of the beginning. Nat Neurosci 2004; $7: 440-5$

Chung JW, Kim GY, Mun YC, Ahn JY, Seong CM, Kim JH. Leukotriene B4 pathway regulates the fate of the hematopoietic stem cells. Exp Mol Med 2005;37:45-50

Crowley-Weber CL, Payne CM, Gleason-Guzman M, Watts GS. Development and molecular characterization of HCT-116 cell lines resistant to the tumor promoter and multiple stress-inducer, deoxycholate. Carcinogenesis 2002;23:2063-80

Elliott ST, Crider DG, Garnham CP, Boheler KR, Van Eyk JE. Two-dimensional gel electrophoresis database of murine R1 embryonic stem cells. Proteomics 2004;4:3813-32

Evans CA, Tonge R, Blinco D, Pierce A, Shaw J, Lu Y, Hamzah HG, Gray A, Downes CP, Gaskell SJ, Spooncer E, Whetton $A D$. Comparative proteomics of primitive hematopoietic cell populations reveals differences in expression of proteins regulating motility. Blood 2004;103:3751-9

Feldmann RE Jr, Bieback K, Maurer MH, Kalenka A, Bürgers HF, Gross B, Hunzinger C, Klüter H, Kuschinsky W, Eichler $\mathrm{H}$. Stem cell proteomes: a profile of human mesenchymal stem cells derived from umbilical cord blood. Electrophoresis 2005;26:2749-58

Gurok U, Steinhoff C, Lipkowitz B, Ropers HH, Scharff C, Nuber UA. Gene expression changes in the course of neural progenitor cell differentiation. J Neurosci 2004;24:5982-02

Gygi SP, Rist B, Gerber SA, Turecek F, Gelb MH, Aebersold R. Quantitative analysis of complex protein mixtures using isotope-coded affinity tags. Nat Biotechnol 1999;17:994-9

Hayman MW, Przyborski SA. Proteomic identification of biomarkers expressed by human pluripotent stem cells. Biochem Biophys Res Commun 2004;316:918-23

Hoffrogge R, Mikkat S, Scharf C, Beyer S, Christoph H, Pahnke J, Mix E, Berth M, Uhrmacher A, Zubrzycki IZ, Miljan $E$, Völker $U$, Rolfs A. 2-DE proteome analysis of a proliferating and differentiating human neuronal stem cell line (ReNcell VM). Proteomics 2006;6:1833-47

Hood L. Systems biology: integrating technology, biology, and computation. Mech Ageing Dev 2003;124:9-16

Husi H, Grant SG. Proteomics of the nervous system. Trends Neurosci 2001;24:259-66

Kim SI, Vöshol H, van Oostrum J, Hastings TG. Neuroproteomics: expression profiling of the brain's proteomes in health and disease. Neurochem Res 2004;29:1317-31

Kwak DH, Yu K, Kim SM, Lee DH, Kim SM, Jung JU, Seo JW, Kim N, Lee S, Jung KY, You HK, Kim HA, Choo YK. Dynamic changes of gangliosides expression during the differentiation of embryonic and mesenchymal stem cells into neural 
cells. Exp Mol Med 2006;38:668-76

Lee HK, Lee BH, Park SA, Kim CW. The proteomic analysis of an adipocyte differentiated from human mesenchymal stem cells using two-dimensional gel electrophoresis. Proteomics 2006;6:1223-9

Lee $\mathrm{YH}$, Han $\mathrm{H}$, Chang SB, Lee SW. Isotope-coded Nterminal sulfonation of peptides allows quantitative proteomic analysis with increased de novo peptide sequencing capability. Rapid Commun Mass Spectrom 2004;18:301927

Levchenko A. Proteomics takes stem cell analyses to another level. Nat Biotechnol 2005;23:828-30

Lubec G, Krapfenbauer K, Fountoulakis M. Proteomics in brain research: potentials and limitations. Prog Neuro-biol 2003;69:193-211

Ma L, Sun B, Hood L, Tian Q. Molecular profiling of stem cells. Clinica Chimica Acta 2007;378:24-32

Maurer MH, Feldmann RE Jr, Futterer CD, Kuschinsky W. The proteome of neural stem cells from adult rat hippocampus. Proteome Sci 2003;1:1-4

Miyachi K, Fritzler MJ, Tan EM. Autoantibody to a nuclear antigen in proliferating cells. J Immunol 2005;121:2228-34

Mueller SM, Glowacki J. Age-related decline in the osteogenic potential of human bone marrow cells cultured in three-dimensional collagen sponges. J Cell Biochem 2001; 82:583-90

Nagano K, Taoka M, Yamauchi Y, Itagaki C, Shinkawa T, Nunomura K, Okamura N, Takahashi N, Izumi T, Isobe T. Large-scale identification of proteins expressed in mouse embryonic stem cells. Proteomics 2005;5:1346-61

Nuttall ME, Gimble JM. Is there a therapeutic opportunity to either prevent or treat osteopenic disorders by inhibiting marrow adipogenesis? Bone 2000;27:177-84

Ong SE, Blagoev B, Kratchmarova I, Kristensen DB, Steen $\mathrm{H}$, Pandey A, Mann M. Stable isotope labeling by amino acids in cell culture, SILAC, as a simple and accurate approach to expression proteomics. Mol Cell Proteomics 2002;1:376-86

Pahnke J, Mix E, Knoblich R, Müller J, Zschiesche M, Schubert B, Koczan D, Bauer P, Böttcher T, Thiesen HJ, Lazarov L, Wree A, Rolfs A. Overexpression of glial cell line-derived neurotrophic factor induces genes regulating migration and differentiation of neuronal progenitor cells. Exp Cell Res 2004;297:484-94

Pazman C, Castelli JC, Wen X, Somogyi R. Large-scale identification of differentially expressed genes during neurogenesis. Neuroreport 2000;11:719-24
Pearce A, Svendsen CN. Characterisation of stem cell expression using two-dimensional electrophoresis. Electrophoresis 1999;20:969-70

Prosperi MT, Ferbus D, Rouillard D, Goubin G. The pag gene product, a physiological inhibitor of c-abl tyrosine kinase, is overexpressed in cells entering $S$ phase and by contact with agents inducing oxidative stress. FEBS Lett 1998;423:39-44

Rohlff C. Proteomics in molecular medicine: applications in central nervous systems disorders. Electrophoresis 2000; 21:1227-34

Rohlff C. Proteomics in neuropsychiatric disorders. Int $\mathrm{J}$ Neuropsychopharmacol 2001;4:93-102

Ross PL, Huang YN, Marchese JN, Williamson B, Parker K, Hattan S, Khainovski N, Pillai S, Dey S, Daniels S, Purkayastha S, Juhasz P, Martin S, Bartlet-Jones M, He F, Jacobson A, Pappin DJ. Multiplexed protein quantitation in Saccharomyces cerevisiae using amine-reactive isobaric tagging reagents. Mol Cell Proteomics 2004;3:1154-69

Sekiya I, Larson BL, Vuoristo JT, Cui JG, Prockop DJ. Adipogenic differentiation of human adult stem cells from bone marrow stroma (MSCs). J Bone Miner Res 2004;19:256-64

Seshi B. An integrated approach to mapping the proteome of the human bone marrow stromal cell. Proteomics 2006;6:5169-82

Shapland C, Hsuan JJ, Totty NF, Lawson D. Purification and properties of transgelin: a transformation and shape change sensitive actin-gelling protein. J Cell Biol 1993;121:1065-73

Stenderup K, Justesen J, Clausen C, Kassem M. Aging is associated with decreased maximal life span and accelerated senescence of bone marrow stromal cells. Bone 2003;33:919-26

Stewart II, Thomson T, Figeys D. 180 labeling: a tool for proteomics. Rapid Commun Mass Spectrom 2001;15:245665

Takasaki Y, Fishwild D, Tan EM. Characterization of proliferating cell nuclear antigen recognized by autoantibodies in lupus sera. J Exp Med 1984;159:981-92

Taylor CM, Marta CB, Claycomb RJ, Han DK, Rasband MN, Coetzee T, Pfeiffer SE. Proteomic mapping provides powerful insights into functional myelin biology. Proc Nat Acad Sci USA 2004;101:4643-8

Wang D, Park JS, Chu JS, Krakowski A, Luo K, Chen DJ, Li $S$. Proteomic profiling of bone marrow mesenchymal stem cells upon transforming growth factor beta1 stimulation. J Biol Chem 2004;279:43725-34 